\title{
Communication \\ SARS-CoV-2 Serology Monitoring of a Cancer Center Staff in the Pandemic Most Infected Italian Region
}

\author{
Chiara Maura Ciniselli ${ }^{1,+}$, Arianna Micali ${ }^{2,+}$, Loris De Cecco ${ }^{\circledR}$, Paola Notti ${ }^{3}$, Valentina Sinno ${ }^{4}$, Elena Luison ${ }^{5}$, \\ Cecilia C. Melani ${ }^{6}$, Maria Grazia Daidone ${ }^{5}\left[\begin{array}{l}\text {, Giovanni Apolone } \\ \end{array}\right.$
}

1 Unit of Bioinformatics and Biostatistics, Department of Applied Research and Technological Development, Fondazione IRCCS Istituto Nazionale dei Tumori (INT), 20133 Milan, Italy; chiara.ciniselli@istitutotumori.mi.it

2 Integrated Biology Platform, Department of Applied Research and Technology Development, Fondazione IRCCS Istituto Nazionale dei Tumori (INT), 20133 Milan, Italy; arianna.micali@istitutotumori.mi.it (A.M.); loris.dececco@istitutotumori.mi.it (L.D.C.)

3 Medical Directorate, Fondazione IRCCS Istituto Nazionale dei Tumori (INT), 20133 Milan, Italy; paola.notti@istitutotumori.mi.it

4 Clinical Trials Center, Fondazione IRCCS Istituto Nazionale dei Tumori (INT), 20133 Milan, Italy; valentina.sinno@istitutotumori.mi.it

5 Biomarker Unit, Department of Applied Research and Technology Development, Fondazione IRCCS Istituto Nazionale dei Tumori (INT), 20133 Milan, Italy; elena.luison@istitutotumori.mi.it (E.L.);

mariagrazia.daidone@istitutotumori.mi.it (M.G.D.); mariangela.figini@istitutotumori.mi.it (M.F.)

6 Scientific Directorate, Fondazione IRCCS Istituto Nazionale dei Tumori (INT), 20133 Milan, Italy; cecilia.melani@istitutotumori.mi.it (C.C.M.); giovanni.apolone@istitutotumori.mi.it (G.A.)

updates

Citation: Ciniselli, C.M.; Micali, A. De Cecco, L.; Notti, P.; Sinno, V.; Luison, E.; Melani, C.C.; Daidone, M.G.; Apolone, G.; Verderio, P.; et al. SARS-CoV-2 Serology Monitoring of a Cancer Center Staff in the Pandemic Most Infected Italian Region. Cancers 2021, 13, 1035. https://doi.org/ 10.3390/cancers13051035

Academic Editors: Gabriella D'Orazi and Mara Cirone

Received: 15 January 2021

Accepted: 24 February 2021

Published: 2 March 2021

Publisher's Note: MDPI stays neutral with regard to jurisdictional claims in published maps and institutional affiliations.

Copyright: (c) 2021 by the authors. Licensee MDPI, Basel, Switzerland. This article is an open access article distributed under the terms and conditions of the Creative Commons Attribution (CC BY) license (https:// creativecommons.org/licenses/by/ $4.0 /)$.
* Correspondence: paolo.verderio@istitutotumori.mi.it; Tel.: +39-02-2390-3201

+ These authors contributed equally to this work.

Simple Summary: Since the beginning of the COVID-19 outbreak, Cancer Centers adopted specific procedures to protect patients as well as to monitor the possible spread of SARS-CoV-2 among healthcare personnel. In April 2020, we implemented a prospective longitudinal study aimed at monitoring the serological response to SARS-Cov-2 in the healthcare personnel of a Comprehensive Cancer Center identified by the Lombardy region (the Italian region most affected by the COVID-19 pandemic) as one of the three oncologic hubs where the Regional Health Authorities referred all the cancer patients in the region. We identified a fraction of healthcare personnel with long-term anti-SARS-CoV-2 antibody response, though negative for viral RNA, who could safely approach fragile cancer patients.

Abstract: Since the beginning of the COVID-19 outbreak, Cancer Centers adopted specific procedures both to protect patients and to monitor the possible spread of SARS-CoV-2 among healthcare personnel (HCP). In April 2020 at Fondazione IRCCS Istituto Nazionale dei Tumori, Milano, one of the three oncologic hubs in Lombardy where the Health Regional Authorities referred all the cancer patients of the region, we implemented a prospective longitudinal study aimed at monitoring the serological response to SARS-Cov-2 in HCP. One hundred and ten HCP answered a questionnaire and were screened by nasopharyngeal swabs as well as for $\operatorname{IgM} / \mathrm{IgG}$ levels; seropositive HCPs were further screened every 40-45 days using SARS-CoV-2-specific serology. We identified a fraction of HCP with long-term anti-SARS-CoV-2 antibody responses, though negative for viral RNA, and thus probably able to safely approach fragile cancer patients. Monitoring asymptomatic HCP might provide useful information to organize the healthcare service in a Cancer Center, while waiting for the effectiveness of the active immunization by SARS-CoV-2 vaccines, which will provide protection from infection.

Keywords: COVID-19; serology; healthcare personnel; comprehensive cancer center; antibody response 


\section{Introduction}

Several recently published papers $[1,2]$ reported contrasting results about the longevity of antibody titers to SARS-CoV-2 and, in general, there is no full agreement in literature on this issue. Although results from some authors seem to support rapid waning of virus-specific IgG antibodies up to approximately three months after infection [3,4], others detected substantial stable titer levels detected over several weeks or several months [5-8].

Since the beginning of the COVID-19 outbreak, Cancer Centers have adopted specific procedures to protect patients, as well as to monitor the possible spread of SARS-CoV-2 among healthcare personnel (HCP) $[9,10]$. At the Fondazione IRCCS Istituto Nazionale Tumori (INT) of Milan, a Comprehensive Cancer Center that became one of the three hubs for oncologic patient referral in Lombardy (the Italian region most affected by the COVID19 pandemic) we implemented in April 2020 a prospective longitudinal study aimed at: (a) screening HCPs without overt symptoms by nasopharyngeal swabs (NPS) and (b) monitoring their IgM/IgG levels every $40-45$ days by SARS-CoV-2-specific serology to assess the persistence of humoral immunity, in combination with the possible occurrence of COVID-19 symptoms as recorded by a questionnaire.

Monitoring $\mathrm{HCP}$ without overt symptoms during the first and second wave of pandemic in Lombardy proved useful to organize the healthcare service in our Cancer Center and will be helpful to test the effectiveness of the SARS-CoV-2 vaccination.

\section{Materials and Methods}

$\mathrm{HCP}$ were prospectively recruited in order to obtain a representative sample of the different professional categories at the INT. All participants signed an informed consent, and the study was reviewed and approved by the Internal Review Board and Ethics Committee.

Between 9th and 29th April, $110 \mathrm{HCP}$ answered a questionnaire and were sampled by venipuncture and NPS. HCP seropositive at admission $\left(\mathrm{T}_{0}\right)$ underwent three additional blood samplings on 11th June ( $\left.\mathrm{T}_{1}\right)$, 22nd-23rd July $\left(\mathrm{T}_{2}\right)$ and 7-9th September $\left(\mathrm{T}_{3}\right)$.

Viral RNA was extracted from nasopharyngeal swabs (Virus RNA Collection kit, LCMGenect, Italy) using DSP Virus/Pathogen midi kit automated on QIAsymphony instrument (Qiagen, Germany). SARS-CoV-2 positivity was investigated in all cases at $\mathrm{T}_{0}$ by real-time polymerase chain reaction (RT-PCR) for N, ORF1ab and E mRNA. In detail, ORF1ab and $\mathrm{N}$ genes were detected using the VIASURE SARS-CoV-2 RT-qPCR (CerTest-Biotec, Spain) following the manufacturer's instructions. The Gene E probe set was synthesized by Integrated DNA Technologies (IDT, TEMAricerca, Italy) based on Corman et al. [11] and detected following the Centre for Disease Control and Prevention (CDC, Atlanta, GA, USA) protocol. Samples were run in duplicate and the data were collected by QuantStudio $12 \mathrm{~K}$ Flex software v1.2.3 (ThermoFisher, Carlsbad, CA, USA). Positivity for the presence of viral RNA in the clinical specimens was established when at least two genes were detected at $\mathrm{Ct}<38$.

Plasma samples were challenged for anti-SARS-CoV-2 IgM/IgG by Enzyme-Linked Immunosorbent Assay (ELISA) supplemented by a confirmatory test for IgG specificity to the immunodominant antigens, Nucleocapsid (NC), and Spike protein, S1 and S2 subunits (Diagnostic Bioprobes, Dia.Pro, Sesto San Giovanni, Milan, Italy) [12] according to the manufacturer's recommendations. The assay was developed with $3,3^{\prime}, 5,5^{\prime}-$ tetramethylbenzidine (TMB) and readings at $450 \mathrm{~nm} / 620-630 \mathrm{~nm}$ after blocking with sulfuric acid. One blank, two negative, and one positive control were included in each test. Results (S/Co) were calculated by dividing the Optical Density (OD) value of the sample by the cut-off value determined with the following formula, based on the mean of the negative controls $\left(\mathrm{N}_{\mathrm{C}}\right)$ : Cut-off $(\mathrm{Co})=\mathrm{N}_{\mathrm{C}}+0.25$. A lateral flow immunochromatographic test (PRIMA LAB SA, Switzerland) and the ChemiLuminescence ImmunoAssay (CLIA) DiaSorin test (DiaSorin, Saluggia, Italy; FDA-EUA) were also applied on plasma samples at $\mathrm{T}_{0}$.

All data were recorded in a dedicated electronic-Case Report Form (e-CRF). Subject characteristics of the cohort were summarized using basic descriptive statistics. Assay data 
were firstly interpreted qualitatively according to the manufacturer's recommendations, then the time trends profiles of the seropositive $\mathrm{HCP}$ at $\mathrm{T}_{0}$ were assessed by resorting to mixed models by considering, as the pivotal variable, the Dia.Pro S/Co values (on logarithmic scale) as a function of time [13]. In these models, the most appropriate matrix of variance-covariance was selected according to the Akaike Information Criterion (AIC). Statistical analyses were carried out with SAS software (Version 9.4; SAS Institute, Inc., Cary, NC, USA) and graphical representations were performed with R-software (ggplot2 package).

\section{Results}

The $110 \mathrm{HCP}$ ranged from 23 to 69 years old (median: $49 \mathrm{yrs)} \mathrm{and} \mathrm{55 \%} \mathrm{were} \mathrm{female;}$ physicians represented $40 \%$ of the study sample, nurses $29 \%$, health workers $25 \%$, administrative personnel $2 \%$ and laboratory personnel or other $4 \%$. A close contact with a confirmed or putative COVID-19-affected case was notified by $54 \%$ of HCP ( $14 \%$ outside INT and $83 \%$ within INT's premises). Among the $110 \mathrm{HCP} 60 \%$ reported no symptoms, $24 \%$ only $1-2$ mild symptoms and the remaining $16 \%$ declared at least three symptoms (headache $72 \%$, fatigue/malaise $66 \%$, myalgia $61 \%$, arthralgia $56 \%$, sore throat $50 \%$, rhinorrhea $38 \%$ ).

All but two subjects were negative for the RT-PCR analysis; the two suspicious cases revealed $\mathrm{Ct} \leq 37$ for the $\mathrm{N}$ gene, $\mathrm{Ct} \leq 34$ or $<38$ for the $\mathrm{E}$ gene and absence of detection for ORF1ab. At $\mathrm{T}_{0}, 21 / 110 \mathrm{HCP}$ had anti-SARS-CoV-2 IgG (19\%) and seven had IgM $(6 \%)$ antibodies, six of whom were also IgG positive according to the Dia.Pro ELISA assay. Among IgG-positive subjects, the confirmation ELISA detected anti-NC in 19 cases out of 21, and anti-S1 and anti-S2 in eight and three cases respectively: IgG specificity for all of the three antigens was detected only in $3 / 21 \mathrm{HCPs}$. The two cases with uncertain RT-PCR values proved to be positive for IgG to NC and $\mathrm{S} 1$ at $\mathrm{T}_{0}$. The subjects positive for $\mathrm{NC}$ and spike were also found positive with PRIMA and DiaSorin (Table 1).

Table 1. Performance at $\mathrm{T}_{0}$ of the Dia.Pro immunoassay (total IgG and confirmation test) compared to alternative approaches.

\begin{tabular}{|c|c|c|c|c|}
\hline \multicolumn{5}{|c|}{ Dia.Pro Immunoreactivity by ELISA (Number of Cases) } \\
\hline & \multicolumn{2}{|c|}{ Total IgG ${ }^{a}$} & \multicolumn{2}{|c|}{ Confirmation Test $^{b}$ (on 21 IgG-Positive Cases) } \\
\hline & Negative $(n=89)$ & Positive $(n=21)$ & NC $(n=19)$ & $\mathrm{S} 1 / \mathrm{S} 2(n=8)$ \\
\hline \multicolumn{5}{|c|}{ PRIMA Test } \\
\hline Negative & 88 & $14(0)$ & 12 & 1 \\
\hline Positive & 1 & $7(2)$ & 7 & 7 \\
\hline \multicolumn{5}{|c|}{ DiaSorin Test $^{c}$} \\
\hline Negative & 88 & $16(0)$ & 14 & 3 \\
\hline Positive & 1 & $5(2)$ & 5 & 5 \\
\hline
\end{tabular}

${ }^{a}$ Dia.Pro cut-off for positivity: $\geq 0.9 \mathrm{~S} / \mathrm{Co} ;{ }^{b}$ Dia.Pro confirmation test cut-off for positivity: $\geq 1 \mathrm{~S} / \mathrm{Co} ;{ }^{\mathrm{c}}$ DiaSorin cut-off for positivity:

$\geq 12$ units; in brackets the number of suspicious cases according to real-time polymerase chain reaction (RT-PCR).

Twenty-two out of the $110 \mathrm{HCP}(20 \%)$ resulted seropositive at $\mathrm{T}_{0}$ according to both IgG and IgM Dia.Pro tests. Nineteen accepted to continue the longitudinal study. Only three of them reported at least three symptoms: all reported headache, two also reported fatigue/malaise, myalgia and arthralgia (one also with conjunctivitis) and one augeusia/disgeusia.

Figure 1 depicts the S/Co time trend profiles of IgG, IgM, anti-NC and anti-S1IgG, respectively, by considering only subjects with a positive $\mathrm{T}_{0}$ result $(n=18,6,18$ and 7 for IgG, IgM, anti-NC and anti-S1 IgG, respectively). 

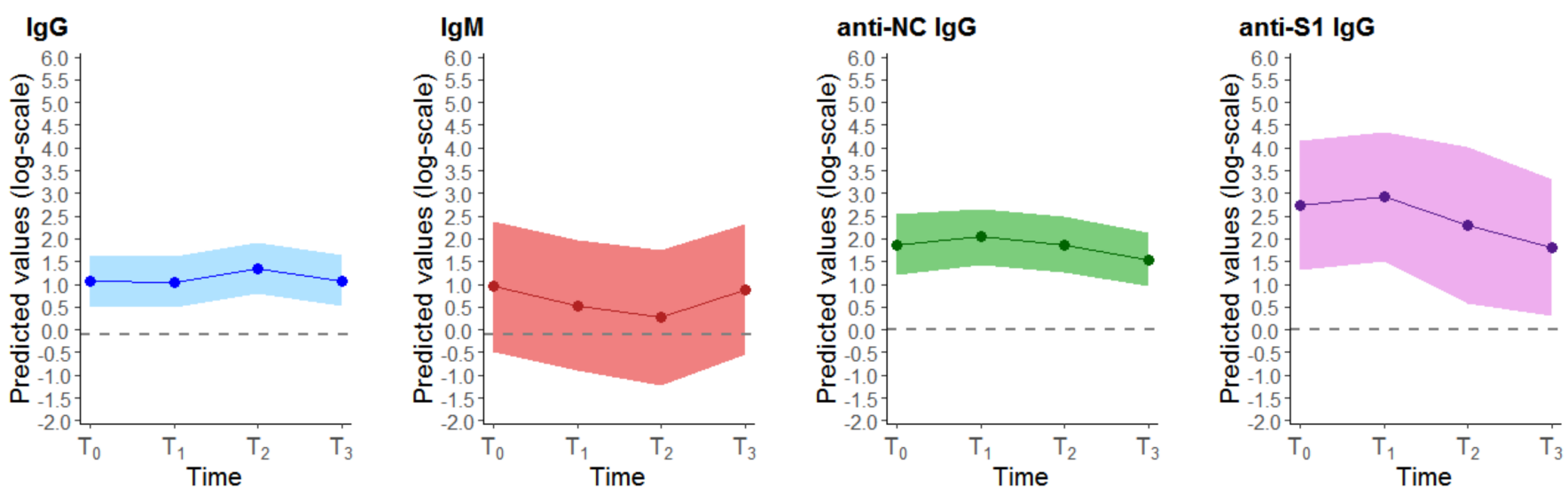

Figure 1. Time trend profiles for the presence of antibodies to SARS-CoV-2 in healthcare personnel with a positive $\mathrm{T}_{0}$ result. Colored dot points indicate the predicted mean value at each time, and the solid lines the estimated time trend together with the $95 \%$ confidence band. The grey dashed line indicates the assay cut-offs for positivity.

\section{Discussion}

In consideration of recurrent COVID-19 pandemic waves and waiting for the effectiveness of anti-SARS-CoV-2 vaccines, our sero-survey was built to monitor putative humoral immunity, not in subjects with overt symptoms but in presumed COVID-19 free HCP unable to comply with the lockdown regulations due to their work duties, and who were in contact with fragile and immunosuppressed oncologic patients more prone to become SARS-CoV-2 targets.

In a relevant proportion of mostly asymptomatic NPS-negative HCP we detected an anti-SARS-CoV-2 seropositivity which persisted after six months with a modest decrease in anti-NC and anti-S1 IgG. This suggests their probable contact with the virus in the past, or at the very beginning of the study, but without overt symptoms in the study time frame. Besides the stability of anti-NC antibodies, it is worth noting that IgG persists to Spike-1, which is proved to correlate with neutralizing antibodies [14].

It is likely that seropositive subjects experienced a mild COVID-19 presentation, since they were enrolled during the peak of the first epidemic wave in Lombardy, the most affected region in Italy. Our sero-surveys, now extended to more HCP, lacked functional assays, although $\mathrm{T}_{0}$ results were confirmed by alternative CE-certified tests, so we cannot infer on the protective activity of these IgG.

To grasp as much information from the data as possible, we also evaluated the S/Co values in the $\mathrm{HCP}$ seropositive at $\mathrm{T}_{0}$ over time by depicting the longitudinal profile of the considered antibodies. In disagreement with some reports [4,7], but in agreement with others [8] on asymptomatic individuals, the length of the immune response in our HCP could be longer than 150 days. We intend to continue our sero-surveys over time to investigate possible protection from reinfection during the new pandemic waves and to provide useful information for the efficacy of SARS-CoV-2 vaccines.

\section{Conclusions}

Monitoring asymptomatic HCP might provide information useful to the organization of the healthcare service in a Cancer Center while waiting for the active immunization by SARS-CoV-2 vaccines, which will provide protection from infection.

Author Contributions: Conceptualization, C.C.M., M.G.D., G.A., P.V. and M.F.; methodology, C.M.C., A.M., L.D.C., E.L., P.V., and M.F.; formal analysis, C.M.C. and P.V.; data curation, A.M., L.D.C., P.N., V.S., E.L., C.C.M., M.G.D. and M.F.; writing-original draft preparation, C.M.C. and A.M.; writingreview and editing, L.D.C., C.C.M., M.G.D., P.V. and M.F.; supervision, G.A.; project administration, C.C.M.; funding acquisition, G.A. All authors have read and agreed to the published version of the manuscript. 
Funding: This research received no external funding.

Institutional Review Board Statement: The study was conducted according to the guidelines of the Declaration of Helsinki and approved by the Institutional Review Board of Fondazione IRCCS Istituto Nazionale dei Tumori-Milano (protocol code INT 65/20, date of approval 3 April 2020).

Informed Consent Statement: Informed consent was obtained from all subjects involved in the study.

Data Availability Statement: The data presented in this study are available on request from the corresponding author.

Acknowledgments: We acknowledge Iolanda Pulice, Clinical Trials Center, Silvia Veneroni, Laura Zanesi, Giuseppe Episcopo, Biomarkers Unit, and Silvia De Sanctis, Scientific Directorate, for clinical study management, subject enrollment, biologic sample collection, preparation and storage; Elena Cavadini, Biomarker Unit, for serologic analysis; Flavio Arienti, Arabella Mazzocchi and Cinzia Biasuz, Transfusion Medicine Unit, for performing and providing Diasorin test results. Giovanni Scoazec for English revision.

Conflicts of Interest: The authors declare no conflict of interest.

\section{References}

1. Röltgen, K.; Powell, A.E.; Wirz, O.F.; Stevens, B.A.; Hogan, C.A.; Najeeb, J.; Hunter, M.; Wang, H.; Sahoo, M.K.; Huang, C.; et al. Defining the Features and Duration of Antibody Responses to SARS-CoV-2 Infection Associated with Disease Severity and Outcome. Sci. Immunol. 2020, 5. [CrossRef] [PubMed]

2. Hartley, G.E.; Edwards, E.S.J.; Aui, P.M.; Varese, N.; Stojanovic, S.; McMahon, J.; Peleg, A.Y.; Boo, I.; Drummer, H.E.; Hogarth, P.M.; et al. Rapid Generation of Durable B Cell Memory to SARS-CoV-2 Spike and Nucleocapsid Proteins in COVID-19 and Convalescence. Sci. Immunol. 2020, 5. [CrossRef] [PubMed]

3. Ibarrondo, F.J.; Fulcher, J.A.; Goodman-Meza, D.; Elliott, J.; Hofmann, C.; Hausner, M.A.; Ferbas, K.G.; Tobin, N.H.; Aldrovandi, G.M.; Yang, O.O. Rapid Decay of Anti-SARS-CoV-2 Antibodies in Persons with Mild Covid-19. N. Engl. J. Med. 2020, 383, 1085-1087. [CrossRef] [PubMed]

4. Long, Q.-X.; Tang, X.-J.; Shi, Q.-L.; Li, Q.; Deng, H.-J.; Yuan, J.; Hu, J.-L.; Xu, W.; Zhang, Y.; Lv, F.-J.; et al. Clinical and Immunological Assessment of Asymptomatic SARS-CoV-2 Infections. Nat. Med. 2020, 26, 1200-1204. [CrossRef] [PubMed]

5. Gudbjartsson, D.F.; Norddahl, G.L.; Melsted, P.; Gunnarsdottir, K.; Holm, H.; Eythorsson, E.; Arnthorsson, A.O.; Helgason, D.; Bjarnadottir, K.; Ingvarsson, R.F.; et al. Humoral Immune Response to SARS-CoV-2 in Iceland. N. Engl. J. Med. 2020, 383, 1724-1734. [CrossRef] [PubMed]

6. Isho, B.; Abe, K.T.; Zuo, M.; Jamal, A.J.; Rathod, B.; Wang, J.H.; Li, Z.; Chao, G.; Rojas, O.L.; Bang, Y.M.; et al. Persistence of Serum and Saliva Antibody Responses to SARS-CoV-2 Spike Antigens in COVID-19 Patients. Sci. Immunol. 2020, 5. [CrossRef]

7. Behrens, G.M.N.; Cossmann, A.; Stankov, M.V.; Schulte, B.; Streeck, H.; Förster, R.; Bosnjak, B.; Willenzon, S.; Boeck, A.-L.; Thu Tran, A.; et al. Strategic Anti-SARS-CoV-2 Serology Testing in a Low Prevalence Setting: The COVID-19 Contact (CoCo) Study in Healthcare Professionals. Infect. Dis. Ther. 2020, 9, 837-849. [CrossRef] [PubMed]

8. Wajnberg, A.; Amanat, F.; Firpo, A.; Altman, D.R.; Bailey, M.J.; Mansour, M.; McMahon, M.; Meade, P.; Mendu, D.R.; Muellers, K.; et al. Robust Neutralizing Antibodies to SARS-CoV-2 Infection Persist for Months. Science 2020, 370, 1227-1230. [CrossRef] [PubMed]

9. Al-Shamsi, H.O.; Alhazzani, W.; Alhuraiji, A.; Coomes, E.A.; Chemaly, R.F.; Almuhanna, M.; Wolff, R.A.; Ibrahim, N.K.; Chua, M.L.K.; Hotte, S.J.; et al. A Practical Approach to the Management of Cancer Patients during the Novel Coronavirus Disease 2019 (COVID-19) Pandemic: An International Collaborative Group. Oncologist 2020, 25, e936-e945. [CrossRef] [PubMed]

10. Valenza, F.; Papagni, G.; Marchianò, A.; Daidone, M.G.; DeBraud, F.; Colombo, M.P.; Frignani, A.; Galmozzi, G.; Ladisa, V.; Pruneri, G.; et al. Response of a Comprehensive Cancer Center to the COVID-19 Pandemic: The Experience of the Fondazione IRCCS-Istituto Nazionale Dei Tumori Di Milano. Tumori 2020, 300891620923790. [CrossRef] [PubMed]

11. Corman, V.M.; Landt, O.; Kaiser, M.; Molenkamp, R.; Meijer, A.; Chu, D.K.; Bleicker, T.; Brünink, S.; Schneider, J.; Schmidt, M.L.; et al. Detection of 2019 Novel Coronavirus (2019-NCoV) by Real-Time RT-PCR. Euro Surveill. 2020, 25, 2000045. [CrossRef] [PubMed]

12. Marlet, J.; Petillon, C.; Ragot, E.; Abou El Fattah, Y.; Guillon, A.; Marchand Adam, S.; Lemaignen, A.; Bernard, L.; Desoubeaux, G.; Blasco, H.; et al. Clinical Performance of Four Immunoassays for Antibodies to SARS-CoV-2, Including a Prospective Analysis for the Diagnosis of COVID-19 in a Real-Life Routine Care Setting. J. Clin. Virol. 2020, 132, 104633. [CrossRef] [PubMed]

13. McCulloch, C.E.; Neuhaus, J.M. (Eds.) Generalized Linear Mixed Models; John Wiley \& Sons, Inc.: New York, NY, USA, 2005.

14. Tian, Y.; Lian, C.; Chen, Y.; Wei, D.; Zhang, X.; Ling, Y.; Wang, Y.; Yeap, L.-S. Sensitivity and Specificity of SARS-CoV-2 S1 Subunit in COVID-19 Serology Assays. Cell Discov. 2020, 6, 75. [CrossRef] [PubMed] 